\title{
El papel de los estudiantes de medicina en las pandemias
}

\author{
The role of medical students in pandemics
}

Jesús Reyna-Figueroa', César Alejandro Arce-Salinas², Hilario Martínez-Arredondo ${ }^{3}$ y Rodolfo Lehmann-Mendoza ${ }^{4}$

'Departamento de Enseñanza e Investigación, Hospital Central Sur de Alta Especialidad; Petróleos Mexicanos.

${ }^{2}$ Dirección, Hospital Central Sur de Alta Especialidad; Petróleos Mexicanos.

${ }^{3}$ Gerencia de Prevención Médica, Servicios de Salud de Petróleos Mexicanos.

${ }^{4}$ Subdirección, Servicios de Salud de Petróleos Mexicanos.

Los autores declaran que no existen conflicto de intereses.

No se recibió financiamiento para la elaboración del presente manuscrito.

Recibido: 31 de marzo de 2020 / Aceptado: 17 de junio de 2020

\section{Resumen}

Existe la percepción general de que los estudiantes de medicina deben ayudar en caso de situaciones de desastre o en contingencias epidemiológicas; aunque es posible que no hayan sido debidamente educados para hacerlo. En últimas fechas, la participación de estudiantes es importante en actividades de capacitación de personal, triage, atención de actividades burocráticas, voluntariado; pero no son considerados como una primera línea de atención en los sitios de desastre o de manejo de pacientes. Se revisa en la literatura médica la percepción de la participación de los estudiantes de medicina en desastres y pandemias y se establecen algunas de las necesidades para su capacitación y enseñanza, para que realmente puedan convertirse en una opción en los momentos de crisis.

Palabras clave: Estudiantes de medicina; pandemias; desastres; enseñanza.

\section{Introducción}

$\mathrm{L}$ a Organización Mundial de la Salud (OMS) denomina pandemia a la propagación universal de una enfermedad que ataca a un porcentaje importante de la población ${ }^{1}$. Cuando una emergencia de esas características se presenta, la participación de los trabajadores sanitarios es un factor medular para el control y el manejo de la enfermedad; por tanto, sus acciones deben estar dirigidas a promover, mantener y proteger la salud comunitaria ${ }^{2,3}$.

Es importante que los médicos estén a la vanguardia en el conocimiento de los conceptos de salud pública y sean adecuadamente

\begin{abstract}
There is a general perception that medical students should help in case of disaster situations or epidemiological contingencies; although, they may not have been properly educated to do so. In recent dates, the participation of students is important in staff training activities, triage, care of bureaucratic activities, volunteering; but they are not considered a first line of care at disaster sites or patient management. The perception of the participation of medical students in disasters and pandemics is reviewed in the literature and some of the needs for their training and teaching are established, so that they can truly become an option in times of crisis.

Keywords: Medical students; pandemics; disasters; teaching.
\end{abstract}

entrenados en las habilidades clínicas para identificar síndromes y proponer las intervenciones sanitarias para el control de las epidemias causadas por microorganismos de alto potencial pandémico, como el virus de la influenza, el virus del SARS, el virus de la inmunodeficiencia humana ${ }^{4-6}$ y recientemente por el SARS-CoV- $2^{7}$.

Un grupo involucrado en los sistemas de salud con actividades encaminadas al aprendizaje más que a la resolución de problemas, son los estudiantes de medicina; su participación en situaciones de desastre o en contingencias epidemiológicas es un tópico del que existen referencias contradictorias. Para realizar un ensayo científico con el objetivo de conocer los argumentos y conclusiones relacionados con el

\section{Correspondencia a:}

Jesús Reyna Figueroa

jesusreynaf@gmail.com 
fueran incluidos como sujetos de estudio en investigaciones post pandémicas realizadas en poblaciones separadas y dispares. Como por ejemplo, el estudio que los comparó con escolares navajos, con la intención de evaluar los títulos del anticuerpo inhibidor de la hemaglutinación H2N2 específico en las infecciones clínicas y subclínicas, en un período de tres años ${ }^{12}$.

En el año de 1968, el virus influenza H3N2 provocó un millón de muertes, debido a que muchos países se supusieron mejor preparados, anticiparon la cobertura de médicos enfermos y movilizaron sus reservas de estudiantes de medicina y enfermería ${ }^{13}$. Otros brotes con menor impacto causados por la cepa A H5N1 se documentaron en 1997 y 2003. En Países Bajos (2003) causado por influenza A H7N7 y en Vietnam junto con Tailandia (2004) responsabilidad de la cepa A H5N1. La importancia de estos últimos radica en que por primera vez se documentaron casos de influenza aviar en humanos, lo que obligó a estructurar sistemas de vigilancia buscando monitorear el posible surgimiento de una pandemia con altas cifras de mortalidad. Para estas últimas, la participación de estudiantes fue prácticamente nula ${ }^{14}$.

Para marzo del año 2020, la OMS declaró pandemia a la infección por SARS-CoV-2, causante de la enfermedad COVID-19. Aunque inicialmente muchas escuelas de medicina adoptaron medidas de distanciamiento social y restringieron el acceso de estudiantes de medicina a zonas asistenciales ${ }^{15}$, en el avance de la pandemia muchas regiones afectadas de España, E.U.A., Argentina, Israel e Italia, contrataron y movilizaron un número importante de estudiantes de medicina, a la par que recontrataron a médicos jubilados, como una estrategia para atender la crisis de salud ocasionada por la pandemia. Las actividades encomendadas variaron dependiendo de las necesidades; por ejemplo, en Barcelona, España, encomendaron a los estudiantes el seguimiento de pacientes, la atención de líneas de atención y actividades burocráticas con el fin de liberar de carga de trabajo al personal médico ${ }^{16-18}$. En otros países como Inglaterra, se aceleró la graduación de estudiantes de medicina del último año para integrarlos a la fuerza laboral, aunque el principal temor era que se convirtieran en una fuente innecesaria de trasmisión e incrementara el número de $\operatorname{casos}^{19}$.

\section{Percepción de la preparación de los estudiantes para participar en pandemias}

Contrario a la percepción general que los médicos (incluyendo a los estudiantes de medicina) son profesionales de respuesta rápida e inmediata ante desastres y pandemias, las conclusiones plasmadas en diferentes revistas científicas establecen que su preparación no les permite ser considerados como parte del personal que 
puede enfrentarlos y no son educados para situaciones de desastre, a pesar de la alta voluntad que ellos puedan tener de responder a la necesidad ${ }^{20,21}$.

Los factores que señalan a los estudiantes como candidatos no adecuados para participar en la primera línea de acción ante contingencias, han sido descritos en la literatura médica. Inicialmente, su categoría de "alumnos" implica la necesidad de un maestro o tutor que guíe sus acciones y que le permita aprender el arte médico y la toma de decisiones; aunado a ello, se ha documentado que a pesar de tener un alto riesgo de infección debido a actividades relacionadas con su formación médica, muchos de los estudiantes no cumplen con las precauciones recomendadas para el control de infecciones; la aceptación de estrategias de prevención suele ser parcial. Por ejemplo, durante la pandemia de influenza A (H1N1) en 2010, su adhesión a la vacunación fue de $91 \%$; sin embargo, la cobertura para la temporada de 2011 cayó a $42 \%$. Los argumentos principales para este incumplimiento fueron: la falta de tiempo, el olvido, el miedo a los efectos secundarios, la falta de información sobre las vacunas, la falta del riesgo percibido y las molestias en el sitio de aplicación; incluso, $61 \%$ eligió seguir asistiendo a clases aun estando enfermos ${ }^{22-25}$.

Otro ejemplo se documenta en el trabajo diario; evaluaciones han revelado que el nivel de conocimiento de los estudiantes (incluyendo a los médicos residentes) con respecto a las precauciones universales, para prevenir infecciones en un hospital es de $100 \%$, aunque el cumplimiento observado es de $89 \%$, lo que los hace poco confiables en la ejecución y uso de barreras de protección ante una contingencia ${ }^{26-29,30}$. Estas cifras traspoladas en la actual pandemia de COVID-19, hacen que los estudiantes de medicina sean más susceptibles a ser infectados por el virus $^{31}$. Desafortunadamente, a diferencia de otras situaciones que requieren un incremento de atención médica, como en desastres naturales donde pueden aprender, en esta pandemia (COVID-19) los estudiantes pueden actuar como agentes de transmisión asintomáticos, además de disminuir los insumos de protección personal y las pruebas diagnósticas disponibles en caso de que enfermen ${ }^{32}$.

Contrariamente a lo anterior, existen reportes de países europeos donde los estudiantes son desplegados en la atención de víctimas y son considerados piedras angulares en la atención de primera línea en una pandemia ${ }^{20}$. Los niveles de educación y entrenamiento pueden ser la diferencia; un estudio realizado en Bélgica, en el que compararon la educación y preparación de estudiantes de medicina de instituciones civiles contra estudiantes de medicina con entrenamiento militar, encontraron que $95 \%$ de los estudiantes de medicina militares recibieron formación en materia radioactivos y nuclear y $22 \%$ participó en otras alternativas para su formación; $44 \%$ percibió que es absolutamente necesario que la educación en desastres debe incorporarse al plan de estudios ordinario, cifras muy por arriba de los encontrado en estudiantes civiles, por lo que el trasfondo militar y el entrenamiento de estos estudiantes los hace, probablemente, mejor preparados para estas situaciones que sus contrapartes civiles ${ }^{33}$.

La reducción de personal sanitario por enfermedad o cansancio, durante desastres o epidemias puede necesitar incluir estudiantes voluntarios, cuya percepción hasta en $77 \%$, es que tienen una obligación moral/ética/profesional de apoyar en estas emergencias; aunque $49 \%$ supone que no tiene que sustituir a la falta de trabajadores y $83 \%$ que su papel debe estar relacionado con el trabajo hospitalario y no al administrativo ${ }^{34,35}$. Particularmente para COVID-19, un estudio reciente encontró un alto nivel de conocimiento relacionado con COVID-19 y comportamientos preventivos autoinformados y una percepción de riesgo moderada entre los estudiantes de medicina iraníes ${ }^{30}$.

\section{Percepción de las instituciones formadoras o comunidad}

Aunque la formación de los futuros médicos en medicina de desastres y las cuestiones de salud pública han sido reconocidas como un componente importante de educación médica de postgrado, los estudiantes de medicina reciben una exposición relativamente limitada a estos temas. El 17,2\% de los estudiantes considera que reciben educación y capacitación adecuadas para enfrentar desastres naturales, $26,2 \%$ para la gripe pandémica y $13,4 \%$ para eventos radioactivos, respectivamente. El $51,6 \%$ considera que está suficientemente capacitado para responder a un desastre natural, $53,2 \%$ ante la gripe pandémica, y $30,8 \%$ para eventos radioactivos. A pesar de la voluntad de los futuros médicos de responder, la educación y la formación en medicina de desastres y preparación para la salud pública es inadecuada ${ }^{21,36,37}$.

Por ello, las universidades, han mostrado la necesidad de discutir las lecciones aprendidas en pasadas pandemias y sugieren añadir ejercicios en escenarios realistas a su programa de salud pública ${ }^{38}$. El conocimiento hace que la parte científica y académica que representa el gremio médico sea un arma contra los problemas sanitarios que pueden generarse, y no pueden darse el lujo de abonar con desinformación al pánico poblacional que suele presentarse en una pandemia.

\section{Roles que pudieran cumplir los estudiantes en situaciones de pandemia}

Basados en la experiencia de pandemias previas, autoridades regulatorias internacionales como la OMS ge- 
neraron rápidamente las recomendaciones para el personal de salud y la población general con el objetivo de controlar el incremento de casos, como el uso de cubrebocas (o de mascarilla N95 para el personal de salud involucrado en la atención de pacientes), el uso de alcohol gel, evitar saludar de mano y de beso, estornudar tapando nariz y boca con el pliegue del codo; el uso de sitios especiales para la revisión de pacientes con síntomas respiratorios y clasificación de los casos, se basaron en las definiciones operativas para el diagnóstico y flujogramas de manejo de la infección ${ }^{39-41}$. Es en la capacitación y difusión de esas acciones de prevención donde los estudiantes de medicina y de enfermería pueden participan activamente, existiendo experiencias de éxito reportadas cuando, a su vez, han recibido una capacitación previa ${ }^{42}$.

Una pandemia puede causar escasez de profesionales sanitarios debido a la enfermedad y una mayor demanda de sus servicios; en consecuencia, muchos países elaboran planes para hacer frente a las crisis sanitarias, y los estudiantes de medicina podrían desempeñar un papel importante. Muy probablemente, un vez que la ciencia de los desastres ha evolucionado, la capacitación del estudiante debe enfocarse en el sistema del triage de desastres. El ordenar, evaluar y clasificar de manera adecuada puede ayudar a salvar muchas vidas ${ }^{43}$.

La enseñanza de la medicina de desastres y del manejo de pandemias requiere de una serie de políticas basadas en procesos que busquen aclarar muchas de las dudas que los médicos de las diferentes instituciones y de los diferentes niveles de atención tienen para una mejora en la atención y que puede basarse en los siguientes puntos:
- Actualizar la epidemiología mundial constantemente, considerando microorganismos re-emergentes.

- Validar los programas universitarios de las diferentes instituciones, requiriendo un mínimo de competencias a aprender por los alumnos.

- Favorecer el aprendizaje por medio de talleres y simulacros.

- Formar grupos de investigación que impulsen el conocimiento de los alumnos de la medicina de desastres y de la salud pública.

- Facilitar herramientas metodológicas y técnicas de enseñanza que permitan disminuir desventajas entre instituciones y centros de atención médica.

- Fomentar la integración de grupos de trabajo, con el compromiso de difundir el conocimiento generado.

- Evaluar periódicamente el impacto de las estrategias, modificando de acuerdo con resultados.

\section{Conclusiones}

La participación de estudiantes de medicina en la primera línea de atención en pandemias y desastres pudiera considerarse una falla en la planeación de las medidas de contingencia y mitigación. Su participación voluntaria supone un acto por el interés de ayudar, pero no hay oportunidad de conocer su real capacidad de respuesta en el momento de la crisis. Probablemente en la participación de atención de llamadas, triage y capacitación del resto de población, pudieran ser las actividades más adecuadas para un grupo que pudiera tener el compromiso moral de ayudar, pero no una preparación adecuada.

\section{Referencias bibliográficas}

1.- Organización Mundial de la Salud. ¿Qué es una pandemia? Disponible en: https://www. who.int/csr/disease/swineflu/frequently_asked_ questions/pandemic/es/

2.- Walsh K, Roma E, Hilton A. Medical education and support for healthcare professionals to prevent future pandemics: report of a conference workshop Ulster Med J 2018; 87 (3): 194-6. PMID: 30559545.

3.- Kumar S, Preetha G. Health promotion: an effective tool for global health. Indian J Community Med 2012; 37 (1): 5-12. doi: 10.4103/0970-0218.94009

4.- Li Y, Wang H, Jin XR, Li X, Pender M, Song CP, et al. Experiences and challenges in the health protection of medical teams in the Chinese Ebola treatment center, Liberia: a qualitative study. Infect Dis Poverty 2018; 7 (92): 1-12. doi: 10.1186/s40249-0180468-6.

5.- Valencia M. Aportes de los nuevos enfoques para la conformación de la salud pública alternativa. Rev Fac Nac Salud Pública 2011; 29 (1): 85-93. http://www.scielo.org.co/pdf/ rfnsp/v29n1/v29n1a10.pdf.

6.- Morsess M, Woolhouse M, Parris C, Carroll D, Karesh WB, Zanbrana C, et al. Prediction and prevention of the next pandemic zoonosis. Lancet 2012; 380: 1956-65. doi: 10.1016/ S0140-6736(12)61684-5.

7.- $\quad$ Lai C C, Shih T P, Ko W C, Tang H J, Hsueh $\mathrm{P}$ R. Severe acute respiratory syndrome coronavirus 2 (SARS-CoV-2) and coronavirus disease-2019 (COVID-19): The epidemic and the challenges. Int J Antimicrob Agents. 2020; 55 (3): 105924. doi: 10.1016/j. ijantimicag.2020.105924.

8.- Ledezma P A. Realidad histórica y metáfora política en Tucídides: la descripción de la "peste" en "La Guerra del Peloponeso" Tesis Doctoral, Madrid España 2011. Disponible en https://eprints.ucm.es/13198/1/T32817.pdf.

9.- Laval E. El Almanaque Americano, la viruela en Santiago en 1872 y los lazaretos.
Rev Chilena Infectol 2015; 32 (2): 227-9. http://dx.doi.org/10.4067/S071610182015000300013.

10.- Martini M, Gazzaniga V, Bragazzi N, Barberis L. The Spanish influenza pandemic: a lesson from history 100 years after 1918. J Prev Med Hyg. 2019; 60: e64-e67. doi: 10.15167/24214248/jpmh2019.60.1.1205.

11.- Trilla A, Trilla G, Daer C. The 1918 "Spanish Flu" in Spain. Clinl Infect Dis 2008; 47: 66873. https://doi.org/10.1086/590567.

12.- Kilbourne E D. Influenza pandemics of the 20th century. Emerg Infect Dis 2006;12 (1): 9-14. doi: 10.3201/eid1201.051254.

13.- Jackson C. History lesson: the Asian Flu pandemic. Br J Gen Pract. 2009; 59(565): 6223. doi: 10.3399/bjgp09X453882.

14.- Tognotti E. Lessons from the history of quarantine, from plague to influenza. Emerg Infect Dis 2013; 19 (2): 254-9. doi: 10.3201/ eid1902.120312.

15.- Rose $\mathrm{S}$. Medical student education in the time of COVID-19 JAMA. 2020 Mar 31. 
doi: 10.1001/jama.2020.5227. [Epub ahead of print].

16.- Jorro I. Cataluña moviliza a los estudiantes de Medicina contra el coronavirus La Crónica Global Disponible en: https://cronicaglobal. elespanol.com/vida/estudiantes-medicinacoronavirus_327213_102.html.

17.- El mañana. Coronavirus: Nueva York llama a estudiantes de medicina para hacer frente a pandemia. Disponible en: https://elmanana. com.mx/coronavirus-nueva-york-llama-aestudiantes-de-medicina-para-hacer-frente-apandemia/).

18.- Amezcua M. Estudiantes de medicina enfrentan al coronavirus sin protocolos, denuncian. El Universal Disponible en: https://www. eluniversal.com.mx/nacion/estudiantesde-medicina-enfrentan-al-coronavirus-sinprotocolos-den.

19.- Lacobucci G. Covid-19: medical schools are urged to fast-track final year students. Br Med J 2020; 368:m1064. doi: 10.1136/bmj.m1064.

20.- Mortelmans L J, Bouman S J, Gaakeer M I, Dieltiens G, Anseeuw K, Sabbe M B. Dutch senior medical students and disaster medicine: a national survey. Int J Emerg Med 2015; 8 (1): 34. doi: 10.1186/s12245-015-0077-0.

21.- Pfeiffer J K. Is the debate and "pause" on experiments that alter pathogens with pandemic potential influencing future plans of graduate students and postdoctoral fellows? mBio 2015; 1 (6): e02525. doi: 10.1128/mbio.02525-14.

22.- Paula S I, Paula G I, Cunegundes K S, MoraesPinto M I. Adherence to influenza vaccination among medical students during and after influenza A (H1N1) pandemic. Rev Inst Med Trop Sao Paulo 2016; 58: 82. doi: 10.1590/ S1678-9946201658082.

23.- May L, Katz R, Johnston L, Sanza M, Bruno C. Assessing physicians' in training attitudes and behaviors during the $2009 \mathrm{H} 1 \mathrm{~N} 1$ influenza season: a cross-sectional survey of medical students and residents in an urban academic setting. Influenza Other Resp Viruses, 2010; 4 (5): 267-75. doi: 10.1111/j.17502659.2010.00151.x.

24.- Lee S I, Aung E M, Chin I S, Hing J W, Mummadi S, Palaniandy G D, et al. Factors affecting medical students' uptake of the 2009 pandemic influenza A (H1N1) vaccine. Influenza Res Treat. 2012; 2012: 753164. doi: 10.1155/2012/753164.

25.- Mak K K, Yiu Y F, Ko K L, Hui K S, Mak K M, Mak L Y, et al. Attitudes and perceptions of influenza vaccination among Hong Kong doctors and medical students before the 2009 pandemic. Eur J Public Health. 2013; 23 (2): 257-62. doi: 10.1093/eurpub/cks014. Epub 2012 Mar 1.

26.- Helfgott A W, Taylor B J, Garcini F J, Eriksen N L, Grimes R. Compliance with universal precautions: knowledge and behavior of residents and students in a Department of Obstetrics and Gynecology. Infect Dis Obstet Gynecol. 1998; 6 (3): 123-8. doi: 10.1002/ (SICI) 1098-0997(1998)6:3<123::AIDIDOG5 $>3.0$.

27.- Kanjirath P P, Coplen A E, Chapman J C, Peters M C, Inglehart M R. Effectiveness of gloves and infection control in dentistry: student and provider perspectives. J Dent Educ. 2009; 73 (5): 571-80. PMID: 19433532.

28.- Basurrah M M, Madani T A. Handwashing and gloving practice among health care workers in medical and surgical wards in a tertiary care center in Riyadh, Saudi Arabia. Scand J Infect Dis. 2006; 38 (8): 620-4. doi: 10.1080/00365540600617025.

29.- Hsu L Y, Jin J, Ang B S, Kurup A, Tambyah P A. Hand hygiene and infection control survey pre-and peri-H1N1-2009 pandemic: knowledge and perceptions of final year medical students in Singapore. Singapore Med J. 2011; 52 (7): 486-90. PMID: 21808958.

30.- May L, Katz R, Johnston L, Sanza M, Petinaux B. Assessing physicians' in training attitudes and behaviors during the $2009 \mathrm{H} 1 \mathrm{~N} 1$ influenza season: a cross-sectional survey of medical students and residents in an urban academic setting. Influenza Other Respir Viruses. 2010; 4 (5): 267-75. doi: 10.1111/j.17502659.2010.00151.x

31.- Taghrir M H, Borazjani R, Shiraly R. COVID-19 and Iranian medical students; a survey on their related-knowledge, preventive behaviors and risk perception. Arch Iran Med. 2020; 23 (4): 249-54. doi: 10.34172/ aim.2020.06.

32.- Barajas O A, Andrade R J, Ramos S V. Retos para la educación médica en México en los tiempos del COVID-19. Gac Med Mex (ahead of Print) disponible en: http:// gacetamedicademexico.com/files/es/ gmm_161_20_retos_barajas_ce_es_20200513 v2.pdf

33.- Mortelmans L J, Lievers J, Dieltiens G, Sabbe M B. Are Belgian military students in medical sciences better educated in disaster medicine than their civilian colleagues? J R Army Med Corps 2016; 162 (5): 383-6. doi: 10.1136/ jramc-2015-000563

34.- Huapaya J A, Maquera-Afaray J, García
P J, Cárcamo C, Cieza J A. Knowledge, practices and attitudes toward volunteer work in an influenza pandemic: cross-sectional study with Peruvian medical students. Medwave. 2015; 15(4): e6136. doi: 10.5867/ medwave.2015.04.6136.

35.- Herman B, Rosychuk R J, Bailey T, Lake R, Yonge O, Marrie T J. Medical students and pandemic influenza Emerg Infect Dis. 2007; 13 (11): 1781-3. doi: 10.3201/ eid1311.070279.

36.- Kaiser H E, Barnett D J, Hsu E B, Kirsch T D, James J J, Subbarao I. Perspectives of future physicians on disaster medicine and public health preparedness: challenges of building a capable and sustainable auxiliary medical workforce. Disaster Med Public Health Prep. 2009; 3 (4): 210-6. doi: 10.1097/ DMP.0b013e3181aa242a

37.- Ozer A, Kirecci E, Ekerbicer H C, Celik M. Medical faculty and school of health student knowledge of and behavior regarding swine flu and vaccine, in Kahramanmaras, Turkey. Southeast Asian J Trop Med Public Health. 2011; 42 (1): 161-7. PMID: 21323179.

38.- Carney J K, Schilling L M, Frank S H, Biddinger P D, Bertsch T F, Grace C J, et al. Planning and incorporating public health preparedness into the medical curriculum. Am J Prev Med 2011; 41 (4 Suppl 3): S193-9. doi: 10.1016/j.amepre.2011.05.026.

39.- Centers for Disease Control and Prevention. Interin guidance on case definition to be used for investigations of novel influenza A (H1N1) cases. 2010. Disponible en: http://www.cdc. gov/h1n1flu/casedef.html.

40.- Secretaria de Salud Guía de manejo de influenza A (H1N1) Rev Fac Med UNAM. 2010; 53 (2): 69-75. http://www.ejournal.unam. $\mathrm{mx} / \mathrm{rfm} / \mathrm{no53}-2 / \mathrm{RFM}$ 053000204.pdf.

41.- The Members of the Novel Swine-Origin Influenza A (H1N1) Virus Investigation. (2009) Emergence of a novel swine-origin influenza A (H1N1) virus in humans. N Engl J Med 2009; 360: 2605-15. doi: 10.1056/NEJMoa0903810.

42.- Vomero A, Delfino M, Pérez P, Pérez F, Pinchak C, Montano A. Experiencia docente en la comunidad sobre pandemia de influenza desarrollada con estudiantes de medicina. Arch Pediatr Urug 2011; 82 (2): 104-7. http://www. scielo.edu.uy/pdf/adp/v82n2/v82n2a08.pdf.

43.- Fink B N, Rega P P, Sexton M E, Wishner C. START versus SALT Triage: which is preferred by the 21 st century health care student? Prehosp Disaster Med. 2018; 33 (4): 381-6. doi: $10.1017 / \mathrm{S} 1049023 \mathrm{X} 18000547$. 